\title{
Atrial Electromechanical Coupling in Patients with Lichen Planus
}

\author{
Mehmet Yaman, MD¹, Uğur Arslan, MD¹, Osman Beton, MD², Lale Dinç Asarcıklı, MD², Aytekin Aksakal, MD', \\ and Orhan Dogdu, MD \\ ${ }^{1}$ Department of Cardiology, Samsun Education and Research Hospital, Samsun, ${ }^{2}$ Department of Cardiology, Diskapi Education and Research Hospital, Ankara, ${ }^{3}$ Department \\ of Cardiology, Firat University, Faculty of Medicine, Elazig, Turkey
}

Background and objectives: A chronic inflammatory disease, lichen planus may cause disturbance of atrial electromechanical coupling and increase the risk of atrial fibrillation. The aim of this study was to evaluate atrial electromechanical delay with both electrocardiography (ECG) and echocardiography in patients with lichen planus (LP).

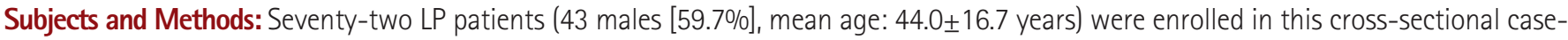
control study. The control group was selected in a 1:1 ratio from 70 patients in an age and sex matched manner. P wave dispersion was measured by ECG to show atrial electromechanical delay. All of the patients underwent transthoracic echocardiography for measuring inter- and intra-atrial electromechanical delays.

Results: The baseline characteristics of the patients and the control group were similar except for the presence of LP. P-wave dispersion measured by ECG was significantly higher in patients with LP ( $p<0.001)$. Patients with LP had significantly prolonged intra- and interatrial electromechanical delays when compared to the control group $(p<0.001)$. In addition, all of these variables were significantly correlated with high sensitive C-reactive protein (hsCRP) levels.

Conclusion: Atrial electromechanical coupling, which is significantly correlated with increased hsCRP levels, is impaired in patients with LP. (Korean Circ J 2016;46(4):530-535)

KEY WORDS: Lichen planus; Echocardiography; Electrocardiography.

\section{Introduction}

Lichen planus (LP) is a chronic inflammatory disease, which affects the skin, genitalia, mucous membranes, and their extensions.) Although the etiology and pathogenesis of this entity are still not fully clarified, it is widely believed that impairment in the T-cell mediated inflammatory response plays a role in its pathogenesis. ${ }^{2)}$

Received: September 23, 2015

Revision Received: November 15, 2015

Accepted: December 1, 2015

Correspondence: Uğur Arslan, MD, Department of Cardiology, Samsun Education and Research Hospital, Baris Bulvari No: 199 Ilkadim-Samsun, Turkey

Tel: 90-532-6039983, Fax: 90-362-2778569

E-mail: ugurarslan5@yahoo.com

- The authors have no financial conflicts of interest.

This is an Open Access article distributed under the terms of the Creative Commons Attribution Non-Commercial License (http://creativecommons. org/licenses/ by-nc/3.0) which permits unrestricted non-commercial use, distribution, and reproduction in any medium, provided the original work is properly cited.
The inflammatory setting observed in LP may cause an increase in the serum triglyceride and low density lipoprotein (LDL) cholesterol levels and a decrease in the high density lipoprotein (HDL) cholesterol levels. ${ }^{2)}$

The frequency of cardiovascular disease is higher in patients with $L P$, which is believed to be due to chronic inflammation, disorders in lipid metabolism, and increased oxidative stress. ${ }^{2)}$ Recent studies have demonstrated an increased risk of atrial fibrillation (AF) in the presence of chronic inflammatory diseases (e.g., psoriasis, rheumatoid arthritis)..-5) There are several clinical, electrocardiographic, laboratory and echocardiographic predictors for the development of AF. P-wave dispersion (PWD) in electrocardiography and atrial electrocardiographic delay measured by echocardiography are the latest promising markers. ${ }^{67)}$

Intra-atrial and inter-atrial conduction times in patients with LP have been assessed based on only PWD on electrocardiography up to now. ${ }^{8)}$ However, studies which enrolled patients with isolated atrial fibrillation and paroxysmal atrial fibrillation revealed that, measurement of echocardiographic delay was more useful than electrocardiography measurement of PWD in the prediction 
of atrial fibrillation. ${ }^{9)}$ In this study, we aimed to evaluate, for the first time, intra-atrial and inter-atrial conduction times with both electrocardiographic PWD and echocardiographic electromechanical delay in patients with LP.

\section{Subjects and Methods}

\section{Study population}

In this study, we enrolled 72 consecutive patients from two different centers who visited the Dermatology Clinic with the diagnosis of LP. The control group was selected in a 1:1 ratio from 70 patients in an age and sex matched manner. The criteria for exclusion in the patient group included the following: any kind of inflammatory diseases, chronic obstructive pulmonary disease, coronary artery disease, valvular heart disease, cardiomyopathy, pulmonary hypertension, abnormal serum electrolyte values, treatment with an antiarrhythmic drug, prior pacemaker implantation, bundle branch block or atrioventricular or intraventricular conduction abnormalities on electrocardiogram (ECG), history of permanent or paroxysmal atrial fibrillation, pericarditis, chronic systemic diseases including renal failure, anemia, thyroid disease, poor echocardiographic imaging quality, and/or presence of infectious disease within 1 week of laboratory and echocardiographic assessment. All the patients were in sinus rhythm. The participants' weights and heights were measured, and their body mass indexes $\left(\mathrm{kg} / \mathrm{m}^{2}\right)$ were calculated. Their blood pressures were measured after a 5-minute rest and again after 10 minutes, and the average of the two measurements was recorded. Two-dimensional and Doppler echocardiographic measurements were performed. Serum triglycerides, LDL-cholesterol, glucose levels, and high sensitive C-reactive protein (hsCRP) levels were assessed in samples collected between 8 a.m. and 9 a.m. after a 12-hour fasting period. The study conformed to the standards set by the Declaration of Helsinki, and ethical approval was obtained from the Medical Ethical Committee of Firat University (Reference No: 02/12/2014-20-22). Written informed consent was obtained from each participant.

\section{Electrocardiographic analysis}

All subjects underwent 12-lead ECG recording (Nihon Kohden Corporation, Tokyo, Japan) after a 20-min resting period in the supine position at a paper speed and amplitude of $50 \mathrm{~mm} / \mathrm{s}$ and 2 $\mathrm{mV} / \mathrm{cm}$, respectively. The P-wave duration was measured manually by using calipers and a 10X magnifying lens for evaluation of the electrocardiographic deflections in all simultaneously recorded 12 leads of the surface ECG by two investigators who were blinded to the participants. In each lead, the mean values for the three complexes were calculated. The onset of the P-wave was defined as the junction between the isoelectric line and the beginning of P-wave deflection and the offset of the P-wave as the junction between the end of the P-wave deflection and the isoelectric line. The $P_{\max }$ measured in any of the 12 leads of the surface ECG was considered to be the longest atrial conduction time. The difference between the maximum $\left(P_{\max }\right)$ and minimum $\left(P_{\text {min }}\right) P$-wave durations was calculated and it was defined as $\mathrm{P}$-wave dispersion $\left(\mathrm{Pd}=\mathrm{P}_{\max }-\right.$ $P_{\text {min }}$. In order to improve accuracy, all measurements were performed with calipers and a magnifying lens to define ECG deflections, as described previously. ${ }^{10)}$

\section{Echocardiographic analysis}

\section{Conventional echocardiography}

The echocardiographic examination was performed at rest, with the patient in the left lateral decubitus position, using an ECG machine (iE33 xMatrix Echocardiography System, Philips, New York, NY, USA) equipped with a 2-4 MHz probe. This test was performed by two experienced echocardiographers, who were blinded to the clinical data. For each measurement, the mean of at least three consecutive cardiac cycles was considered. Left ventricular enddiastolic, left ventricular end-systolic, and left atrial end-systolic diameters were measured in $\mathrm{M}$-mode in the parasternal longaxis views according to the standards of the American Society of Echocardiography."1) Left ventricular ejection fraction was calculated via a modified biplane Simpson's method from apical four- and two-chamber views. Left and right atrial areas were calculated from the apical chamber view. ${ }^{11)}$ Right ventricular diameter and tricuspid annular plane systolic excursion (TAPSE) measurements were made in M-mode according to the guidelines. ${ }^{12)}$ From the suprasternal window, color M-mode Doppler imaging was performed with the cursor parallel to blood flow in the descending aorta. The color Doppler Nyquist limit was set to $30-50 \mathrm{~cm} / \mathrm{s}$, switching to the M-mode with a recorder sweep rate of $200 \mathrm{~mm} / \mathrm{s}$. When the isovelocity slope was unclear, the baseline aliasing velocity was changed. Aortic propagation velocity was calculated by dividing the distance between points corresponding to the beginning and end of the propagation slope by the duration between these two points. Then, the mean of the three calculations was considered. ${ }^{13)}$ Carotid intima media thickness (CIMT) was measured with 7-MHz transducer attached to the available machine (Vivid S5, GE Vingmed Ultrasound AS, Hortan, Norway). Images were obtained bilaterally from the distal part of the common carotid artery. The two bright echogenic lines in the arterial wall were defined as the intimamedia lines. At the end-diastole (peak of the R wave on ECG), CIMT was measured between these two lines, and the average of the 
three measurements was taken.13)

\section{Atrial electromechanical coupling}

Continuous ECG recording was performed from a single lead. Tissue Doppler echocardiography was performed using transducer frequencies of 3.5-4.0 MHz, adjusting the spectral pulsed Doppler signal filters until a Nyquist limit of $15-20 \mathrm{~cm} / \mathrm{sec}$ was achieved. The monitor sweep speed was set at $50-100 \mathrm{~mm} / \mathrm{sec}$ to optimize the spectral display of myocardial velocities. In the apical four-chamber view, the pulsed Doppler sample volume was subsequently placed at the level of left ventricular lateral mitral annulus, septal mitral annulus, and right ventricular tricuspid annulus. Tissue Doppler pattern was characterized by a positive (i.e., above baseline) myocardial systolic wave (S) and two negative (i.e., below baseline) diastolic waves-early (E) and atrial (A). Efforts were made to align the pulsed wave cursor in order to maintain the Doppler angle of incidence as close to zero as possible in relation to the direction of these walls. E deceleration time and isovolumetric relaxation time (IVRT) were measured. IVRT was defined as the interval between the end of the aortic outflow and the beginning of the mitral inflow signal. Atrioventricular annulus motions were recorded simultaneously with electrocardiogram in lead II during sinus rhythm to analyze the temporal relationship between atrial electrical phases and myocardial motion. The intervals from the

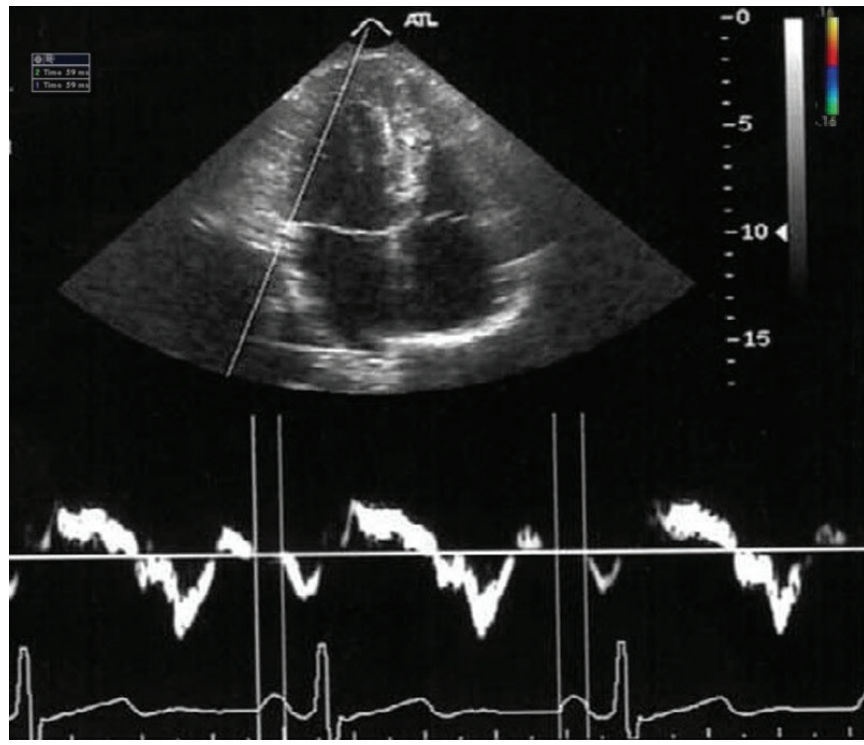

Fig. 1. Measurement of the PA interval with tissue Doppler imaging, which denotes time interval from the onset of $P$ wave on the surface electrocardiogram to the beginning of the late diastolic wave ( $A_{m}$ wave). PA: P-wave to the beginning of A-wave on surface ECG.

Table 1. Demographic, clinical, and laboratory data of the study population

\begin{tabular}{|c|c|c|c|}
\hline & $\begin{array}{l}\text { LP patients } \\
\quad(n=72)\end{array}$ & $\begin{array}{c}\text { Control group } \\
(n=70)\end{array}$ & $\mathbf{p}$ \\
\hline Age (years) & $44.0 \pm 16.7$ & $41.7 \pm 13.7$ & 0.38 \\
\hline Males & $43(59.7)$ & 37 (52.9) & 0.41 \\
\hline Smoking & $31(43.1)$ & $28(40)$ & 0.71 \\
\hline Hypertension & $6(8.3)$ & $3(4.3)$ & 0.26 \\
\hline Diabetes mellitus & $2(2.8)$ & $1(1.4)$ & 0.51 \\
\hline $\mathrm{BMI}\left(\mathrm{kg} / \mathrm{m}^{2}\right)$ & $26.0 \pm 4.7$ & $27.3 \pm 4.1$ & 0.13 \\
\hline Fasting glucose (mg/dL) & $87.9 \pm 6.6$ & $85.9 \pm 7.6$ & 0.52 \\
\hline $\mathrm{LDL}-\mathrm{C}(\mathrm{mg} / \mathrm{dL})$ & $128.3 \pm 19.4$ & $107.4 \pm 25.3$ & $<0.001$ \\
\hline Triglycerides (mg/dL) & $221.1 \pm 76.9$ & $152.2 \pm 47.0$ & $<0.001$ \\
\hline $\mathrm{HDL}-\mathrm{C}(\mathrm{mg} / \mathrm{dL})$ & $35.0 \pm 4.3$ & $42.4 \pm 9.4$ & $<0.001$ \\
\hline hsCRP (mg/L) & $5.3 \pm 1.9$ & $2.2 \pm 1.0$ & $<0.001$ \\
\hline Creatinine (mg/dL) & $0.8 \pm 0.2$ & $0.8 \pm 0.1$ & 0.94 \\
\hline Systolic BP $(\mathrm{mmHg})^{*}$ & $123.1 \pm 15.5$ & $126.8 \pm 15.0$ & 0.27 \\
\hline Diastolic BP $(\mathrm{mmHg})^{*}$ & $74.9 \pm 9.8$ & $75.8 \pm 9.7$ & 0.63 \\
\hline Heart rate $(\mathrm{bpm})^{*}$ & $73.2 \pm 11.6$ & $71.7 \pm 9.9$ & 0.55 \\
\hline Oral steroid use & $8(11.1)$ & 0 & 0.004 \\
\hline Statin use & $5(6.9)$ & $7(10.0 \%)$ & 0.51 \\
\hline
\end{tabular}

Data are expressed as $n(\%)$ or mean \pm standard deviation. ${ }^{*} \mathrm{BP}$ and heart rate measurements were obtained at the time of echocardiographic examination. LP: lichen planus, BMI: body mass index, LDL-C: low-density lipoprotein cholesterol, HDL-C: high density lipoprotein cholesterol, hsCRP: high sensitive Creactive protein, BP: blood pressure 
Table 2. Echocardiographic measurements of the study population

\begin{tabular}{|c|c|c|c|}
\hline & $\begin{array}{l}\text { LP patients } \\
\quad(n=72)\end{array}$ & $\begin{array}{l}\text { Control group } \\
\qquad(n=70)\end{array}$ & $\mathbf{p}$ \\
\hline \multicolumn{4}{|l|}{ 2D echocardiography } \\
\hline LVEDD (mm) & $48.2 \pm 4.0$ & $47.6 \pm 3.9$ & 0.39 \\
\hline $\operatorname{LVESD}(\mathrm{mm})$ & $29.0 \pm 3.8$ & $28.9 \pm 3.2$ & 0.62 \\
\hline LV ejection fraction (\%) & $67.3 \pm 8.5$ & $67.0 \pm 7.2$ & 0.88 \\
\hline LA diameter (mm) & $33.5 \pm 2.9$ & $34.1 \pm 2.1$ & 0.16 \\
\hline $\mathrm{LA}$ volume index $\left(\mathrm{mL} / \mathrm{m}^{2}\right)$ & $20.3 \pm 4.4$ & $20.1 \pm 4.0$ & 0.64 \\
\hline RA area $\left(\mathrm{cm}^{2}\right)$ & $14.8 \pm 1.4$ & $14.8 \pm 1.5$ & 0.73 \\
\hline RA area index $\left(\mathrm{cm}^{2} / \mathrm{m}^{2}\right)$ & $8.3 \pm 1.2$ & $8.1 \pm 1.0$ & 0.41 \\
\hline Aorta diameter (mm) & $27.0 \pm 2.9$ & $26.9 \pm 3.3$ & 0.91 \\
\hline IVS diameter (mm) & $9.6 \pm 1.0$ & $11.2 \pm 2.1$ & 0.52 \\
\hline PW diameter (mm) & $9.3 \pm 1.1$ & $9.3 \pm 1.4$ & 0.91 \\
\hline TAPSE (mm) & $18.4 \pm 1.8$ & $18.3 \pm 1.8$ & 0.71 \\
\hline Right ventricular diameter (mm) & $24.8 \pm 4.1$ & $24.4 \pm 4.0$ & 0.62 \\
\hline Pulmonary artery systolic pressure $(\mathrm{mmHg})$ & $24.0 \pm 4.0$ & $23.6 \pm 4.0$ & 0.54 \\
\hline LV mass $(\mathrm{g})$ & $101.2 \pm 18.9$ & $100.5 \pm 17.6$ & 0.28 \\
\hline \multicolumn{4}{|l|}{ Doppler echocardiography } \\
\hline $\mathrm{E}(\mathrm{cm} / \mathrm{sec})$ & $82.3 \pm 11.6$ & $80.8 \pm 16.0$ & 0.54 \\
\hline $\mathrm{A}(\mathrm{cm} / \mathrm{sec})$ & $63.6 \pm 13.1$ & $63.5 \pm 13.5$ & 0.96 \\
\hline EDT (msec) & $218.4 \pm 37.7$ & $224.6 \pm 42.5$ & 0.37 \\
\hline IVRT (msec) & $87.1 \pm 19.1$ & $92.0 \pm 21.7$ & 0.16 \\
\hline$E / A$ & $1.3 \pm 0.3$ & $1.3 \pm 0.4$ & 0.89 \\
\hline \multicolumn{4}{|l|}{ Tissue Doppler echocardiography } \\
\hline $\mathrm{Em}(\mathrm{cm} / \mathrm{sec})$ & $10.1 \pm 3.1$ & $11.2 \pm 2.7$ & 0.029 \\
\hline Am $(\mathrm{cm} / \mathrm{sec})$ & $5.7 \pm 2.1$ & $7.6 \pm 1.4$ & $<0.001$ \\
\hline $\mathrm{Sm}(\mathrm{cm} / \mathrm{sec})$ & $11.1 \pm 2.4$ & $11.0 \pm 3.4$ & 0.84 \\
\hline Et $(\mathrm{cm} / \mathrm{sec})$ & $9.6 \pm 3.2$ & $9.4 \pm 2.9$ & 0.32 \\
\hline At $(\mathrm{cm} / \mathrm{sec})$ & $5.4 \pm 1.6$ & $7.3 \pm 1.5$ & $<0.001$ \\
\hline St (cm/sec) & $10.7 \pm 3.2$ & $10.2 \pm 2.7$ & 0.52 \\
\hline Lateral PA (msec) & $74.5 \pm 8.1$ & $59.2 \pm 4.2$ & $<0.001$ \\
\hline Septal PA (msec) & $59.2 \pm 7.2$ & $52.6 \pm 4.9$ & $<0.001$ \\
\hline Tricuspid PA (msec) & $49.3 \pm 4.4$ & $45.9 \pm 3.8$ & $<0.001$ \\
\hline $\begin{array}{l}\text { Inter-atrial delay (msec) } \\
\text { (lateral PA-RV PA) }\end{array}$ & $25.2 \pm 7.5$ & $13.6 \pm 4.7$ & $<0.001$ \\
\hline $\begin{array}{l}\text { Intra-atrial delay (msn) (LA) } \\
\text { [lateral PA-septal PA (intra left atrial mechanical delay)] }\end{array}$ & $15.3 \pm 4.9$ & $7.0 \pm 3.4$ & $<0.001$ \\
\hline $\begin{array}{l}\text { Intra-atrial delay (RA) } \\
\text { [septal PA-RV PA (intra right atrial mechanical delay)] }\end{array}$ & $10.0 \pm 5.8$ & $6.7 \pm 3.1$ & $<0.001$ \\
\hline
\end{tabular}

LP: lichen planus, LVEDD: left ventricle end diastolic diameter, LVESD: left ventricle end-systolic diameter, LV: left ventricle, LA: left atrium, RA: right atrium, IVS: interventricular septum, PW: left ventricular posterior wall, TAPSE: tricuspid annular plane systolic excursion, E: peak mitral valve flow velocity during the early rapid filling phase, A: peak mitral valve flow velocity during atrial contraction, EDT: deceleration time of early phase of mitral valve flow, IVRT: isovolumetric relaxation time, PA: delay from the onset of P-wave on ECG to the onset of A-wave on tissue Doppler recordings, RV: Right ventricle 
Table 3. Comparison of $\mathrm{P}$-wave duration values between the two groups

\begin{tabular}{lccr}
\hline & $\begin{array}{c}\text { LP patients } \\
(\mathbf{n}=\mathbf{7 2})\end{array}$ & $\begin{array}{c}\text { Control group } \\
(\mathbf{n = 7 0 )}\end{array}$ & $\mathbf{p}$ \\
\hline$P_{\max }$ & $98.7 \pm 16.3$ & $87.7 \pm 10.9$ & $<0.001$ \\
$P_{\min }$ & $62.2 \pm 14.2$ & $65.9 \pm 10.4$ & 0.079 (NS) \\
$P W D(m s)$ & $36.5 \pm 7.6$ & $21.9 \pm 5.2$ & $<0.001$ \\
\hline
\end{tabular}

$\mathrm{P}_{\max }$ : maximum $\mathrm{P}$-wave duration, $\mathrm{P}_{\min }$ : minimum $\mathrm{P}$-wave duration, PWD: P-wave dispersion, NS: Non-significant

onset of P-wave on surface ECG to the beginning of A-wave $(P A)$, representing atrial electromechanical delay (Fig. 1) were obtained from lateral mitral annulus, septal mitral annulus, and right ventricular (RV) tricuspid annulus and designated as lateral $P A$, septal $P A$, and $R V P A$, respectively. The timing of mechanical activation of each reference point, namely, lateral mitral, septal mitral, and RV tricuspid annuli depends on the distances of these points from the sinus node. The difference between septal PA and RV PA was defined as intra-right atrial mechanical delay (septal PA-RV PA); the difference between lateral PA and septal PA was defined as intra-left atrial mechanical delay (lateral PA-septal PA); and the difference between lateral PA and RV PA (lateral PA-RV PA) was defined as inter-atrial mechanical delay. ${ }^{14)}$

\section{Statistical analysis}

All statistical analyses were performed by using SPSS package program ver.10.0 (SPSS Inc., Chicago, IL, USA). Continuous variables were reported as means \pm standard deviation and categorical variables were reported as percentages. Student's t-test was used for comparison of normally distributed variables and MannWhitney U-test was used for non-normally distributed variables. Categorical variables were compared by the $\chi^{2}$ test or Fisher's exact test as appropriate. Pearson's correlation analysis was used for correlations. $p$ values less than 0.05 were considered to be statistically significant.

\section{Results}

Seventy-two LP patients were enrolled in the study, of whom 43 were men (59.7\%). The mean age of the patients was $44.0 \pm 16.7$ years. The control group consisted of 70 age and sex matched healthy volunteers. Clinical characteristics and echocardiographic findings of the two groups are summarized in Tables 1 and 2. Age, sex, body mass index, presence of diabetes and hypertension, smoking status, baseline systolic and diastolic blood pressures, heart rate levels, and left ventricular (LV) end-diastolic diameter, LV end-systolic diameter, LV mass, LA diameter, LA volume index, RA area index and LV ejection fraction, and deceleration time of early phase of mitral valve flow and IVRT, and TAPSE and RV diameter were similar between the two groups. However, patients with LP exhibited significantly higher levels of LDL-cholesterol, triglycerides, and hsCRP, and lower levels of HDL-cholesterol. Oral steroid use was also significantly higher in the LP group than in the control group.

Atrial electromechanical delay measurements are shown in Table 2. Patients with LP had significantly prolonged lateral PA, septal PA, tricuspid $\mathrm{PA}_{\mathrm{A}}$ and intra- and interatrial electromechanical delays compared with the control group $(p<0.001)$. The intra-atrial and inter-atrial delays were found to be significantly correlated with hsCRP ( $r=0.60, p<0.001$ and $r=0.58, p<0.001$, respectively).

$P_{\max }$ and $P_{\min }$ values are shown in Table 3. PWD was significantly higher in patients with LP $(p<0.001)$. PWD was also found to be significantly correlated with hsCRP $(r=0.51, p<0.01)$.

\section{Discussion}

In this study, we showed that patients with LP had significantly higher PWD, intra and inter-atrial delays, and higher LDL and hsCRP levels when compared to the healthy controls. LP is a chronic inflammatory autoimmune mucocutaneous disease. Although the exact pathogenesis of the disease is still unclear, autoantibodies and T-cell mediated cytotoxicity have been implicated, ${ }^{15)}$ and most of the cardiovascular disorders share similar pathogenetic mechanisms, such as chronic inflammation, endothelial dysfunction and increased oxidative stress. ${ }^{16)}$

In their review, Michelucci et al ${ }^{17)}$ told that prolonged PWD has a predictive value in the development of AF in patients with or without apparent heart disease under various circumstances. Our results may be important for the early detection of subclinical cardiac involvement in LP patients. Increased hsCRP level and significant correlation between hsCRP levels and PWD in this study indicated that inflammation might be a possible mechanism for increased PWD in LP. Similarly, in another study, hsCRP and PWD were shown to be correlated with each other in LP patients. ${ }^{8)}$ Additionally in our study, we found increased intra-atrial and interatrial delays in patients with $L P$, which were correlated with hsCRP. The echocardiographic measurement of atrial electromechanical 


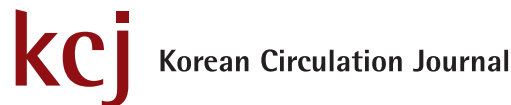

delay has been shown to be correlated with the development of AF in several patient groups. ${ }^{18) 19)}$ Therefore, according to our study results, we can only hypothesize that LP patients with ongoing inflammation might have atrial electro-mechanical delay with a risk of AF development.

The major limitation of our study is its cross-sectional design with lack of follow-up of patients. The number of patients enrolled in our study is also small. As a result, we could not demonstrate whether prolongation of intra and inter atrial electro-mechanical delays predicted AF in LP patients. Another limitation is that we manually calculated P-wave measurements by a magnifying lens as in previous studies, instead of using computer-assisted P-wave calculation methods.

In conclusion, although LP is a disease characterized by increased inflammatory activity, there are no existing clinical data on whether atrial inflammation is higher in these patients. Our study is the first in this regard to suggest that atrial electro-mechanical coupling detected by echocardiography is impaired in concordance with increased inflammatory markers.

\section{References}

1. Arias-Santiago S, Buendía-Eisman A, Aneiros-Fernández J, et al. Lipid levels in patients with lichen planus: a case-control study. J Eur Acad Dermatol Venereo/ 2011;25:1398-401.

2. Arias-Santiago $S$, Buendía-Eisman A, Aneiros-Fernández J, et al. Cardiovascular risk factors in patients with lichen planus. Am J Med 2011;124:543-8.

3. Bacaksiz A, Erdogan E, Tasal A, et al. Electrocardiographic P-wave characteristics in patients with psoriasis vulgaris. Ups J Med Sci 2013;118:35-41.

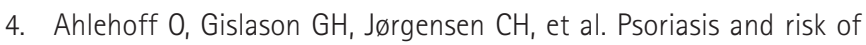
atrial fibrillation and ischaemic stroke: a Danish Nationwide Cohort Study. Eur Heart J 2012;33:2054-64.

5. Acar G, Sayarlioğlu M, Akçay A, et al. Evaluation of atrial electromechanical delay and left atrial mechanical functions in patients with rheumatoid arthritis. Turk Kardiyol Dern Ars 2009;37:447-53.

6. Deniz A, Yavuz B, Aytemir $K_{1}$ et al. Intra-left atrial mechanical delay detected by tissue Doppler echocardiography can be a useful marker for paroxysmal atrial fibrillation. Echocardiography 2009;26:779-84.

7. Deniz $A$, Sahin DY, Kanadasi M, et al. Conduction characteristics in atrial fibrillation. Predictive value of tissue Doppler echocardiography.
Herz 2014;39:137-41.

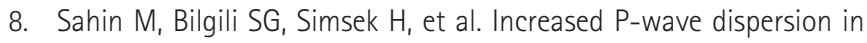
patients with newly diagnosed lichen planus. Clinics (Sao Paulo) 2013;68:846-50.

9. Ari $H_{1}$ Ari $\mathrm{S}, \mathrm{Akkaya} M$, et al. Predictive value of atrial electromechanical delay for atrial fibrillation recurrence. Cardiol J 2013;20:639-47.

10. Simsek H, Gunes Y, Demir C, Sahin M, Gumrukcuoglu HA, Tuncer M. The effects of iron deficiency anemia on $p$ wave duration and dispersion. Clinics (Sao Paulo) 2010;65:1067-71.

11. Lang RM, Bierig M, Devereux RB, et al. Recommendations for chamber quantification: a report from the American Society of Echocardiography's Guidelines and Standards Committee and the Chamber Quantification Writing Group, developed in conjunction with the European Association of Echocardiography, a branch of the European Society of Cardiology. J Am Soc Echocardiogr 2005;18:1440-63.

12. Rudski LG, Lai WW, Afilalo J, et al. Guidelines for the echocardiographic assessment of the right heart in adults: a report from the American Society of Echocardiography endorsed by the European Association of Echocardiography, a registered branch of the European Society of Cardiology, and the Canadian Society of Echocardiography. J Am Soc Echocardiogr 2010;23:685-713.

13. Sahin $M$, Simsek $H$, Akyol $A$, et al. A new echocardiographic parameter of arterial stiffness in end-stage renal disease. Herz 2014;39:749-54.

14. Ozer N, Yavuz B, Can I, et al. Doppler tissue evaluation of intra-atrial and interatrial electromechanical delay and comparison with P-wave dispersion in patients with mitral stenosis. J Am Soc Echocardiogr 2005;18:945-8

15. Middel $P$, Lippert $U$, Hummel KM, et al. Expression of lymphotoxinalpha by keratinocytes: a further mediator for the lichenoid reaction. Pathobiology 2000;68:291-300.

16. Flammer AJ, Ruschitzka F. Psoriasis and atherosclerosis: two plaques, one syndrome? Eur Heart J 2012;33:1989-91.

17. Michelucci A, Bagliani G, Colella A, et al. P wave assessment: state of the art update. Card Electrophysiol Rev 2002;6:215-20.

18. Calık AN, Ozcan KS, Cağdaş M, et al. Electromechanical delay detected by tissue Doppler echocardiography is associated with the frequency of attacks in patients with lone atrial fibrillation. Cardiol $\mathrm{J}$ 2014;21:138-43.

19. Nar G, Inci S, Aksan G, Soylu K, Demirelli S, Nar R. The relationships between atrial electromechanical delay and CHA2DS2-VASc score in patients diagnosed with paroxysmal AF. Echocardiography 2015;32:1359-66. 